\title{
REFLEXIONES SOBRE LA GUÍA BIOÉTICA PARA ASIGNACIÓN DE RECURSOS LIMITADOS DE MEDICINA CRÍTICA EN SITUACIÓN DE EMERGENCIA EN MÉXICO
}

\author{
ADOLFO ESPINOSA DE LOS MONTEROS RODRÍGUEZ \\ adolfo.espinosa@cuc.udg.mx \\ JOSÉ LÓPEZ GUIARTE \\ jose.lguiarte@academicos.udg.mx \\ EDNA RAMÍREZ ROBLES \\ edna.ramirez@academicos.udg.mx
}

Profesores a tiempo completo adscritos al Departamento de Estudios Jurídicos del Centro

Universitario de la Costa de la Universidad de Guadalajara (México)

Fecha de recepción: 01/01/2021 - Fecha de aceptación: 02/02/2021

RESUMEN: El presente artículo es una reflexión sobre las propuestas de Guía bioética para asignación de recursos limitados de medicina crítica en situación de emergencia, emitidas en México. Se expone el tratamiento de acceso a la salud de los adultos mayores en tiempos de COVID-19, en el que se proponen elementos para la construcción de un andamiaje normativo que privilegie la autonomía, la dignidad y el respeto a los derechos humanos de las personas, frente a otras consideraciones instrumentales.

Palabras clave: derecho a la salud, derechos humanos, bioética, triaje, COVID-19, adultos mayores, OMS

ABSTRACT: This article is a reflection on the bioethical guide proposals for the allocation of limited resources for critical medicine in emergency situations issued in Mexico. It presents the access to health treatment of the elderly COVID-19 times. Additionally, it proposes elements to build a normative scaffolding that privileges autonomy, dignity and respect of human rights.

Keywords: right to health, human rights, bioethics, triage, COVID-19, elderly, OMS

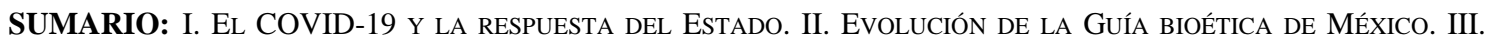
Exhorto de la Comisión Nacional de los Derechos Humanos. IV. Propuesta ante la Cámara de Diputados

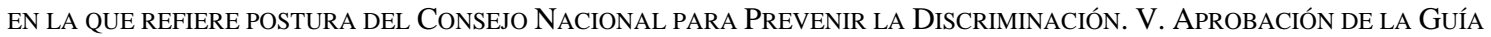
BIOÉTICA PARA ASIGNACIÓN DE RECURSOS LIMITADOS DE MEDICINA CRÍTICA EN SITUACIÓN DE EMERGENCIA. VI. CONCLUSIONES. VII. BibliografíA. VIII. DOCUMENTOS. 


\section{EL COVID-19 Y LA RESPUESTA DEL ESTADO}

Ante la declaración de la pandemia de COVID-19 en el mes de marzo del año en curso, la Organización Mundial de la Salud (OMS), hizo el llamamiento a los países a tomar medidas urgentes y agresivas con el fin de estar a tiempo de cambiar el curso de la pandemia. Lo anterior, impactaría sensiblemente a la sociedad y a su economía; sin embargo, se aclaraba que no se debía de obviar el respeto a los derechos humanos, como se muestra a continuación:

Sabemos que estas medidas están teniendo un gran impacto sobre sus sociedades y sus economías, igual que lo tuvieron en China.

Todos los países deben encontrar un delicado equilibrio entre la protección de la salud, la minimización de los trastornos sociales y económicos, y el respeto de los derechos humanos.

Concluyó la OMS el llamado priorizando los siguientes lineamientos de acción a los países: prevención, preparación, salud pública, liderazgo político y por encima de todo señaló a las personas. En México, en previsión de lo referido por la OMS, y en cumplimiento al contenido de la Constitución Mexicana en materia de salud, se reunió el Consejo de Salubridad General al ser el órgano competente para establecer las medidas preventivas indispensables y obligatorias en todo el país. Constituido en sesión permanente reconoció como epidemia al virus SARS-CoV2 (COVID) declarándola una enfermedad grave de atención prioritaria. Estableció, entre otras, medidas especiales de reconversión hospitalaria y exhortó a las entidades federativas a expandir la capacidad de atención oportuna previendo un incremento en la hospitalización.

\section{EVOLUCIÓN DE LA GUÍA BIOÉTICA DE MÉXICO}

En las primeras semanas del mes de abril del 2020 se publicó en la página oficial del Consejo de Salubridad General la propuesta de la primera guía bioética realizada por un grupo multidisciplinar de expertas y expertos en el tema, titulada Guía bioética para la asignación de recursos limitados de medicina crítica en situación de emergencia ${ }^{1}$. El propio instrumento establece en su preámbulo el contexto en el que debía ser considerada la observación de la guía, que refiere que:

Este documento tiene como propósito ser una guía bioética para la toma de decisiones de triaje cuando una emergencia de salud pública genera una demanda en los recursos de medicina crítica que no es posible satisfacer. Esta guía fue elaborada para hacer frente a la pandemia de COVID19. El COVID-19 es una enfermedad viral infecciosa nueva, causada por el virus SARSCoV-2, que tiene manifestaciones clínicas muy serias en aproximadamente el 5\% de la población que la contrae. Actualmente no existe tratamiento profiláctico ni curativo para el COVID-19. Esta guía únicamente debe de entrar en operación si:

* La capacidad existente de cuidados críticos está sobrepasada, o está cerca de ser sobrepasada, y no es posible referir pacientes que necesitan de cuidados críticos a otros servicios de salud donde puedan ser atendidos de manera adecuada. ${ }^{1}$

Como se advierte en la cita anterior, aplica esta propuesta en la toma de decisiones de triaje en una emergencia de salud pública, cuando se genera demanda de recursos de medicina crítica que no es posible satisfacer. Dicho con otras palabras, en este contexto de emergencia de salud pública aplica cuando la demanda de atención en las unidades de cuidados intensivos UCI- es mayor a los recursos con los que se cuenta. Por ello, la primera propuesta de la guía

\footnotetext{
${ }^{1}$ Guía bioética para asignación de recursos limitados de medicina crítica en situación de emergencia. México. (Primera versión, 13 de abril de 2020) Consejo de Salubridad General. Disponible en https://interactivo.eluniversal.com.mx/online/pdf-20/PDF-GUIA Bioetica FINAL 13 Abril2020.pdf
} 
está dividida en dos partes: la primera está centrada en el aspecto bioético de la asignación de recursos escasos de la medicina crítica; y la segunda, describe el procedimiento a utilizar en la asignación de los recursos. En esta primera propuesta de Guía se plantearon algunos razonamientos como son los siguientes:

En situaciones donde no existe una emergencia de salud pública, la práctica médica cotidiana y la práctica de salud pública operan de manera simultánea, pero con objetivos que pueden ser contrarios. En la práctica médica cotidiana el objetivo primordial es prevenir, tratar o curar las enfermedades o padecimientos de un paciente en especifico. Los principios bioéticos que generalmente se consideran que gobiernan dicha práctica son los de beneficencia, nomaleficencia, autonomía y justicia. En la práctica de salud pública el objetivo primordial es que la salud de la población sea lo mejor posible de acuerdo a la cantidad de recursos disponibles. El principio que generalmente se considera que gobierna a la salud pública es el de la justicia social.

(...) el objetivo de la salud pública durante una emergencia de salud pública es doble: tratar el mayor número de pacientes y salvar la mayor cantidad de vidas. Salvar la mayor cantidad de vidas requiere evaluar: i) la posibilidad de que un paciente mejore y sobreviva, y ii) el tiempo que dicho paciente utilizará los recursos escasos que se pueden utilizar.

Asignar recursos escasos de medicina crítica para salvar el mayor número de vidas durante una emergencia de salud pública genera, al menos dos dilemas. Primero, como asignar recursos escasos cuando dos (o más) pacientes son similares características (i.e. edad, comorbilidades, gravedad de la enfermedad) y pronóstico médico. La manera aceptada de asignar recursos escasos cuando nos encontramos en situaciones donde los pacientes son similares es elecciones al azar. Seleccionar al azar evita que consideraciones impertinentes entren en juego. Segundo, como asignar recursos escasos cuando la prognosis es similar pero la diferencia de edad entre los pacientes es significativa. Por ejemplo, cuando sólo tenemos un ventilador y hay dos pacientes: un paciente A de 80 años y un paciente B de 20 años. Supongamos que si paciente A recibe el ventilador ella vivirá 7 años más y si paciente $B$ recibe el ventilador ella vivirá 65 años más. Para solucionar este problema se tiene que introducir un principio adicional: salvar la mayor cantidad vidas-por-completarse. Una vida por completarse se debe de entender como aquella que aún no ha pasado por los diferentes estados de desarrollo bio-psico-social humanos (i.e. infancia, adolescencia, edad madura, vejez). Y de entre las vidas por completarse hay que elegir aquéllas que están en etapas más tempranas. Ahora, en lugar de realizar distinciones de edad de veta fina (por meses o días, por ejemplo) lo que se propone son las siguientes categorías clasificatorias: 012, 12-40, 41-60, 61-75 y +75 . Utilizar el principio de vidas-por-completarse podría parecer injustamente discriminatorio hacia los adultos mayores, pero no es así. Y para entender el porqué tendrá que remontar a la pregunta: porque la muerte es mala para nosotros. La respuesta a esa pregunta es que la muerte es mala para nosotros porque nos priva de oportunidades valiosas futuras. Ello quiere decir que por lo general la muerte priva a los jóvenes de un número mayor de bienes que a aquellas personas que ya han pasado por dicha etapa vital. Por lo tanto un sistema de asignación de recursos escasos que favorece salvar la mayor cantidad de vidas-porcompletarse es un sistema que prioriza a aquellos que por lo general perderían más si murieran².

La propuesta fue recibida con polémica, tanto por miembros del propio Consejo de Salubridad General, como por expertos y la prensa generalista: "La UNAM se deslinda de guía que recomienda priorizar a jóvenes sobre tercera edad"3, "Guía Bioética, "inadecuada" ante contingencia por Covid-19: experto de la UNAM"4, "Consejo de Salubridad General no aprobó

\footnotetext{
${ }^{2}$ Ibid., pp. 2-3.

${ }^{3}$ MORENO, T., "UNAM se deslinda de guía que recomienda priorizar a jóvenes sobre tercera edad", El Universal, 14 de abril 2020. Disponible en https://www.eluniversal.com.mx/nacion/coronavirus-unam-se-deslinda-de-guia-querecomienda-priorizar-jovenes-sobre-tercera-edad

${ }^{4}$ MORENO, T., "Guía Bioética, "inadecuada" ante contingencia por Covid-19: experto de la UNAM", El Universal, 15 de abril 2020. Disponible en https://www.eluniversal.com.mx/nacion/guia-bioetica-inadecuada-ante-contingenciapor-covid-19-experto-de-la-unam
} 
"Guía bioética" es un borrador" " "Guía Bioética de Medicina Crítica debe ser ampliamente discutida: UNAM", "Bajan a "proyecto" la Guía Bioética para asignar recursos durante Covid$19 " 7$. En medio de la discusión el Consejo de Salubridad General mediante el Comunicado 108/20 $0^{8}$ estableció que se presentaría una propuesta de Guía para la asignación de recursos en situación de contingencia a vocales del Consejo de Salubridad General. Refirió entre otras cosas, que el proyecto tiene el propósito de proveer información actualizada y específica al oficial de triaje para la toma de decisiones y quitar la carga psicológica al personal tratante:

Por lo anterior, el doctor José Ignacio Santos Preciado, secretario del Consejo de Salubridad General, autorizó la publicación en el sitio electrónico del propio Consejo: http://www.csg.gob.mx/ el documento que se denomina PROYECTO DE GUÍA PARA ASIGNACIÓN DE RECURSOS EN SITUACIÓN DE CONTINGENCIA, con el propósito de darlo a conocer para observaciones y comentarios, no solo de la comunidad médica, sino también de la opinión pública.

Los propósitos del proyecto referido son: coadyuvar con la Secretaria de Salud en la Jornada Nacional de Sana Distancia; proveer de información actualizada y especifica al oficial de triaje; facilitar y apoyar al oficial de triaje en el proceso de la toma de decisiones y quitarle la carga psicológica al personal tratante de los enfermos de COVID-19.

Después de la publicación y en medio de la polémica de la Guía presentó renuncia el Comisionado Nacional de Bioética9 ${ }^{9}$ El Secretario del Consejo de Salubridad General, refirió que la: "guía busca salvar vidas y terminar con el amiguismo e influyentismo en la toma de decisiones, por lo cual se establecerá un equipo de Triaje que garantice imparcialidad"10.

\section{EXHORTO DE LA COMISIÓN NACIONAL DE LOS DERECHOS HUMANOS}

La preocupación social por los adultos mayores implicó que la Comisión Nacional de los Derechos Humanos a realizara un exhorto al Consejo de Salubridad General para garantizar los derechos humanos y rechazar medidas discriminatorias, centrándose en las siguientes precisiones sobre la Guía:

- es contraria al derecho a la salud, vinculantes para México, en el sistema universal y regional de protección de los derechos humanos.

\footnotetext{
${ }^{5}$ REDACCIÓN AN, “Consejo de Salubridad General no aprobó "Guía bioética”; es un borrador”, Aristegui Noticias, 15 de abril 2020. Disponible en https://aristeguinoticias.com/1504/mexico/consejo-de-salubridad-general-no-aproboguia-bioetica-es-un-borrador-enterate/

${ }^{6}$ SÁNCHEZ, A., "Guía Bioética de Medicina Crítica debe ser ampliamente discutida: UNAM”, La Jornada, 15 de abril 2020. Disponible en https://www.jornada.com.mx/ultimas/sociedad/2020/04/15/guia-bioetica-de-medicinacritica-debe-ser-ampliamente-discutida-unam-471.html

${ }^{7}$ MIRANDA, P., "Bajan a "proyecto" la Guía Bioética para asignar recursos durante Covid-19", El Universal, 16 de abril 2020. Disponible en https://www.eluniversal.com.mx/nacion/bajan-proyecto-la-guia-bioetica-para-asignarrecursos-durante-covid-19

${ }^{8}$ Comunicado 108/20, Se presentará propuesta de Guía para asignación de recursos en situación de contingencia a vocales del CSG, Secretaría de Salud, Consejo de Salubridad General, 16 de abril 2020. Disponible en http://www.csg.gob.mx/descargas/triaje/Comunicado 108 CSG Guixa Asignacioxn recursos en situacioxn de co ntingencia150420.pdf

9 POY, L., "Manuel Ruiz de Chávez dimite como comisionado nacional de Bioética”, La Jornada, 16 abril 2020. Disponible en https://www.jornada.com.mx/ultimas/politica/2020/04/16/renuncia-el-comisionado-nacional-debioetica-manuel-ruiz-de-chavez-9295.html

10 TORIBIO, L. Y RODRÍGUEZ, P., "Renuncia delegado de Guía de Bioética de COVID-19”, Imagen Radio, 17 de abril 2020. Disponible en https://www.imagenradio.com.mx/renuncia-delegado-de-guia-de-bioetica-de-covid-19
} 
- justifica que el Estado Mexicano no garantice el efectivo acceso a la salud a todos los pacientes en caso de requerir acceso a la medicina crítica.

- ejerce discriminación indirecta hacia las personas mayores, al ponerlos en desventaja en el acceso de medicina crítica.

- en esas condiciones vulnera el derecho a la salud, a la vida y a la integridad personal de las personas mayores.

- que es delicado que se proponga que el triaje funcione como órgano decisorio sin que este acompañado por el Comité de Ética.

- es ilegal al invadir la competencia del Poder Judicial en los términos de la Ley de Amparo.

- contiene criterios e indicaciones contrarios al bloque constitucional de respeto y protección de los derechos humanos de todas las personas sin excepción ${ }^{11}$.

La suma de las precisiones de la Comisión Nacional de los Derechos Humanos advierten la preocupación por el respeto al derecho a la salud de las personas mayores, de ahí el exhorto al Consejo de Salubridad General al "pleno respeto, protección y garantía de los derechos humanos en términos constitucionales, así como de la normatividad aplicable al caso" ${ }^{12}$. De igual forma, emitió un exhorto al presidente de México para la ratificación de la Convención Interamericana sobre la Protección de los Derechos Humanos de las Personas Mayores, adoptada el 15 de junio de 2015, por la Asamblea General de la Organización de los Estados Americanos. Toda vez que dicho instrumento "busca promover, proteger y asegurar el reconocimiento y el pleno goce y ejercicio, en condiciones de igualdad, de todos los derechos humanos y libertades fundamentales de la persona mayor, a fin de contribuir a su plena inclusión, integración y participación en la sociedad"13.

\section{PROPUESTA ANTE LA CÁMARA DE DIPUTADOS EN LA QUE REFIERE POSTURA DEL CONSEJO NACIONAL PARA PREVENIR LA DISCRIMINACIÓN (CONAPRED $)^{14}$}

La propuesta ante la Cámara de Diputados del punto de acuerdo tiene como fin que la Guía Bioética de Asignación de Recursos de Medicina Critica respete el marco constitucional, los tratados internacionales sobre derechos humanos y atienda a las personas hospitalizadas en las unidades de cuidados intensivos por COVID-19. Sometiendo al pleno la propuesta siguiente:

- El rechazo a que la Guía establezca criterios basados en la edad o cualquier tipo de discriminación.

\footnotetext{
${ }^{11}$ COMISIÓN NACIONAL DE LOS DERECHOS HUMANOS, La Comisión Nacional de los Derechos Humanos exhorta al Consejo de Salubridad General a implementar medidas para garantizar los derechos humanos de todas las mexicanas y mexicanos y rechazar medidas discriminatorias, con motivo de la emergencia derivada de la pandemia originada por el coronavirus COVID-19, 17 de abril de 2020. Disponible en https://www.cndh.org.mx/sites/default/files/documentos/2020-04/Com_2020_137_0.pdf

12 Ibid., p. 2.

${ }^{13}$ Ibid., p. 3.

${ }^{14}$ Proposición con punto de acuerdo por la que la Cámara de Diputados del H. Congreso de la Unión rechaza que la Guía bioética de asignación de recursos de medicina crítica, se establezca cualquier tipo de criterio basado en la edad o cualquier tipo de discriminación, para la atención crítica de los enfermos por COVID-19. Disponible en http://gaceta.diputados.gob.mx/PDF/64/2020/abr/PROPOS-28-ABR/Propo-Morena-27.pdf
} 
- El exhorto al Consejo de Salubridad General para que el contenido de la Guía se abstenga de incluir conceptos "que signifique un acto de discriminación y se apegue de manera estricta a la protección y garantía de los derechos humanos en términos Constitucionales, así como de la normatividad aplicable al caso, buscando en tomo momento la preservación del mayor número de vidas posible"15.

- El exhorto al Poder Ejecutivo para la firma y ratificación de la Convención Interamericana sobre la Protección de los Derechos Humanos de las Personas Mayores, adoptada el 15 de junio de 2015, por la Asamblea General de la Organización de los Estados Americanos.

Por lo que se advierte que la propuesta, va muy de la mano con los criterios establecidos por la Comisión Nacional de los Derechos Humanos, en el sentido de no establecer criterios de discriminación por edad, en la prestación del servicio de salud a personas afectadas por el COVID-19; además del exhorto reiterado al presidente de México para la ratificación de instrumentos internacionales específicamente de protección a las personas Mayores. Es de resaltar que en el cuerpo de la propuesta analizada, concretamente en el punto SEXTO, se vierten las observaciones de la Secretaría de Gobernación y el Consejo Nacional para Prevenir la Discriminación (Conapred), misma que refiere a pie de página No. 20. Segob, Conapred. 15 de abril de 2020. Nota sobre la Revisión de la "Guía Bioética de Asignación de Recursos de Medicina Critica" desde el Derecho a la Igualdad y No Discriminación y señala entre los aspectos más destacables:

- Ahora bien, la Guía señala dos conjuntos de criterios que el equipo de triaje deberá aplicar. Por una parte, están los criterios derivados de los exámenes médicos (7 componentes, entre ellos la edad); y, por la otra, los 2 criterios para el desempate de pacientes con puntajes similares, es decir, la edad y el azar. Hay que señalar que, de estos 9 criterios, solo la edad es considerada una categoría sospechosa de discriminación y, como tal, debe ser examinada a la luz de los estándares establecidos como obligatorios por la Suprema Corte de Justicia de la Nación.

- De acuerdo con la jurisprudencia de la SCJN, cualquier medida de diferenciación en el acceso a derechos y oportunidades y que se base en categorías sospechosas de discriminación debe cumplir con tres requisitos fundamentales: 1) cumplir una finalidad imperiosa desde el punto de vista constitucional; 2) estar estrechamente vinculada con la finalidad, y 3) ser la medida menos restrictiva posible para conseguir efectivamente la finalidad.

- El criterio de la edad no cumple con estos requisitos por un igual número de razones: 1) la propia Constitución específica que está prohibido establecer discriminaciones a partir de esta características y que todos los derechos, entre ellos la salud, corresponden a todas y todos los habitantes del territorio nacional sin excepción; 2) proteger la salud de todas las personas no tiene un vínculo de necesidad con la exclusión de algunas de ellas por una característica, como la edad, que pudiera significar menores probabilidades de éxito para el tratamiento, y 3) la exclusión de las personas de mayor edad no es la mejor manera de maximizar los recursos materiales y humanos disponibles durante la emergencia con el propósito de lograr los fines de la salud pública, dado que la experiencia en otros países muestra que las personas mayores pueden tener una condición de salud que les permita recuperarse del COVID-19. ${ }^{16}$ Por todo lo anterior, se considera que la edad debería ser excluida de los criterios médicos y de desempate

\footnotetext{
${ }^{15}$ Ibid., p. 21.

16 SUPREMA CORTE DE JUSTICIA DE LA NACIÓN, Categoría sospechosa, su escrutinio. Tesis: P./J. 10/2016 (10a.). Gaceta del Semanario Judicial de la Federación, Décima Época, Pleno, Libro 34, Septiembre de 2016, Tomo I, Pág. $8 . \quad$ Jurisprudencia (Constitucional). $\quad$ Disponible en https://sjf.scjn.gob.mx/sjfsist/Paginas/DetalleGeneralV2.aspx ?Clase $=$ DetalleTesisBL $\& I D=2012589 \&$ Semanario $=0$
} 
para que las personas puedan acceder a los servicios de cuidado intensivo, escasos como son en época regular y urgentes en contextos de pandemia ${ }^{17}$.

Las recomendaciones establecidas por la CONAPRED consisten en:

- Retirar el criterio médico de la edad para el puntaje asignado en el triaje, así como en el criterio de desempate para acceso al servicio de cuidados intensivos.

- No alarmar a las personas mayores y a sus familias, evitando prejuicios como que sólo las personas mayores se enferman y los jóvenes son invulnerables al COVID-19.

- Desarrollar comunicación eficiente y sencilla sobre los contenidos de la Guía en la población; para cambiar la percepción de que se excluye a las personas mayores. Refiere que: "El derecho a la salud también se integra por estos elementos a los que las personas deben acceder sin discriminación" 18 .

- Desarrollar un mecanismo para asegurarse que no se integre un equipo de triaje reproduzca, en el curso de sus decisiones, prejuicios y prácticas que puedan resultar en atención discriminatoria.

- Que se incluya en las decisiones del triaje a instituciones competentes como la CONAPRED, CNDH, CONAMED, etcétera, en la decisión de los recursos y que los mismos sean del conocimiento público, especialmente a las personas que les afectan.

- Que la discriminación estructural implica que las personas tienen limitado acceso a bienes y servicios que procuran la salud y calidad de vida, por lo que no se debe culpabilizar a las personas. Es necesario que se valore que muchas afectaciones a la salud y comorbilidades de las personas enfermas provienen de contextos de desigualdad estructural, tales como "trastornos alimenticios (desnutrición, obesidad y enfermedades relacionadas) o a la falta de acceso universal a la salud (como ocurre con algunas enfermedades crónicas o formas de discapacidad)" ${ }^{\prime 19}$.

- Que se señale que la Guía es de aplicación excepcional, porque el punto de partida no es discriminar, ni limitar derechos a ningún colectivo.

La propia Cámara de Diputados de la Federación, solicitó que se interviniera en el nuevo proyecto ante el Consejo de Salubridad General. La opinión pública continuó comentando ampliamente el proyecto de Guía bioética; pidiendo información sobre el tratamiento diferenciado a los adultos mayores en el acceso a la atención en las unidades de cuidados intensivos, y el ejercicio del azar en selección del paciente para la asignación de los recursos de medicina crítica.

\section{APROBACIÓN DE LA GUÍA BIOÉTICA PARA ASIGNACIÓN DE RECURSOS LIMITADOS DE MEDICINA CRÍTICA EN SITUACIÓN DE EMERGENCIA}

Posteriormente, a finales de ese mismo mes se aprobó una nueva Guía bioética para asignación de recursos limitados de medicina crítica en situación de emergencia ${ }^{20}$, misma que

\footnotetext{
${ }^{17}$ Op. cit., nota 14, pp. 16-20.

${ }^{18}$ Ibid., p. 19.

${ }^{19}$ Ibid., p. 20.

${ }^{20}$ Guía bioética para asignación de recursos limitados de medicina crítica en situación de emergencia (Versión actual, 30 de abril de 2020), Consejo de Salubridad General. Disponible en http://www.csg.gob.mx/descargas/pdf/index/informacion relevante/GuiaBioeticaTriaje 30 Abril $20207 \mathrm{pm} . \mathrm{pdf}$
} 
incorporó los criterios tanto de la CNDH como los de la CONAPRED. Incluyendo como fundamento los artículos $1^{\circ}$ (igualdad y no discriminación) $)^{21}, 2^{\circ}$ (derechos indígenas a la salud) ${ }^{22}, 4^{\circ}$ (derecho a la salud) ${ }^{23}, 73$ (facultades del Consejo de Salubridad General) ${ }^{24}$ de la Constitución Mexicana, la Ley General de Salud y del Reglamento Interior del Consejo de Salubridad General, del Comité de Ética de la Comisión Consultiva Científica, la resolución 1/2020 de la Comisión Interamericana de los Derechos Humanos ${ }^{25}$.

Establece la Guía en la fracción V de Principios Bioéticos que orientan y justifican la asignación de recursos limitados de medicina crítica en situación de emergencia, en su segundo párrafo que "La guía consta de dos secciones. La primera sección se justifica cierta manera específica de asignar recursos escasos de medicina crítica, sustentándose en los Derechos Humanos y la Bioética"26.

Por lo anterior, se advierte que el peso gravitacional del contenido de la misma se encuentra en el respeto a la dignidad de las personas, a la igualdad y la no discriminación, al respeto a la vida, a la salud y a la integridad física de las personas, así como en los principios de la bioética: beneficencia, no maleficencia, autonomía y justicia. La Guía refiere expresamente lo siguiente:

Durante la designación de recursos escasos de medicina crítica se deben tomar medidas de forma justa y por razones de salud pública sin utilizar criterios que discriminen a las personas. Esto quiere decir que se ha de procurar un reparto justo de los beneficios y las cargas, haciendo un uso eficiente de los recursos disponibles. Las siguientes características no deberán de ser tomadas en

${ }^{21}$ Refiere la Constitución de los Estados Unidos Mexicanos en su Artículo $1^{\text {o }}$ que: "En los Estados Unidos Mexicanos todas las personas gozarán de los derechos humanos reconocidos en esta Constitución y en los tratados internacionales de los que el Estado Mexicano sea parte, así como de las garantías para su protección, cuyo ejercicio no podrá restringirse ni suspenderse, salvo en los casos y bajo las condiciones que esta Constitución establece.

Las normas relativas a los derechos humanos se interpretarán de conformidad con esta Constitución y con los tratados internacionales de la materia favoreciendo en todo tiempo a las personas la protección más amplia.

Todas las autoridades, en el ámbito de sus competencias, tienen la obligación de promover, respetar, proteger y garantizar los derechos humanos de conformidad con los principios de universalidad, interdependencia, indivisibilidad y progresividad. En consecuencia, el Estado deberá prevenir, investigar, sancionar y reparar las violaciones a los derechos humanos, en los términos que establezca la ley.

Está prohibida la esclavitud en los Estados Unidos Mexicanos. Los esclavos del extranjero que entren al territorio nacional alcanzarán, por este solo hecho, su libertad y la protección de las leyes.

Queda prohibida toda discriminación motivada por origen étnico o nacional, el género, la edad, las discapacidades, la condición social, las condiciones de salud, la religión, las opiniones, las preferencias sexuales, el estado civil o cualquier otra que atente contra la dignidad humana y tenga por objeto anular o menoscabar los derechos y libertades de las personas".

${ }^{22}$ Refiere la Constitución de los Estados Unidos Mexicanos en su Artículo 2. Inciso b) fracción III: “Asegurar el acceso efectivo a los servicios de salud mediante la ampliación de la cobertura del sistema nacional, aprovechando debidamente la medicina tradicional, así como apoyar la nutrición de los indígenas mediante programas de alimentación, en especial para la población infantil”.

${ }^{23}$ Refiere la Constitución de los Estados Unidos Mexicanos en su Artículo 4 (cuarto párrafo): "Toda Persona tiene derecho a la protección de la salud. La Ley definirá las bases y modalidades para el acceso a los servicios de salud y establecerá la concurrencia de la Federación y las entidades federativas en materia de salubridad general, conforme a lo que dispone la fracción XVI del artículo 73 de esta Constitución. La Ley definirá un sistema de salud para el bienestar, con el fin de garantizar la extensión progresiva, cuantitativa y cualitativa de los servicios de salud para la atención integral y gratuita de las personas que no cuenten con seguridad social".

24 Op. cit., Nota 2.

${ }^{25}$ CORTE INTERAMERICANA DE LOS DERECHOS HUMANOS, Declaración de la Corte Interamericana de Derechos Humanos 1/20. COVID-19 y Derechos Humanos: Los problemas y desafíos deben ser abordados con perspectivas de Derechos Humanos y Respetando las obligaciones Internacionales, 9 de abril de 2020. Disponible en https://www.corteidh.or.cr/tablas/alerta/comunicado/declaracion_1_20_ESP.pdf

${ }^{26}$ Ibid., p. 2. 
cuenta durante la asignación de recursos escasos de medicina crítica: (...) edad (...). En particular, se debe cuidar que la limitación al derecho social a la salud durante una emergencia de salud pública no castigue más a poblaciones en situación de vulnerabilidad.

* La única característica que debe tomarse en cuenta para ser candidato (a) a la asignación de recursos escasos de medicina crítica es la posibilidad demostrable de acuerdo con la experiencia médica nacional e internacional de beneficiarse de dichos recursos médicos ${ }^{27}$.

La Guía sigue poniendo el énfasis en la no discriminación por la edad de los pacientes que requieran el cumplimiento del derecho a la salud y la atención especializada de medicina crítica:

Esta guía no excluye a ningún paciente que sería, en condiciones ordinarias, sujeto a recibir cuidados críticos. Esto quiere decir, en principio, que la edad cronológica o tener una discapacidad no son de por si características que excluyan a pacientes de ser candidatos (as) a recibir cuidados críticos. Es un error que ha de evitarse a toda costa asumir que tener cierta edad o cualquier discapacidad es sinónimo de tener una calidad de vida inferior, un pronóstico desfavorable, o mala salud ${ }^{28}$.

La Guía de igual forma contempla que se ha de respetar la autonomía del paciente, por lo que deben de ser informados sobre los tratamientos e intervenciones a la que tengan derecho. De igual forma, el respeto de voluntad anticipada, de conformidad a la norma vigente, por lo que "el deseo de no ser sujeto a cierto tratamiento o intervención siempre debe respetarse" 29 . De igual forma contempla el derecho a recibir en su caso cuidados paliativos, y el cumplimiento del deber ético de no abandonar al paciente.

La Guía también refiere que tiene el objetivo de proteger la salud de la población "entendiendo esto como salvar la mayor cantidad de vidas. Lo anterior se traduce en que los pacientes que tienen mayor probabilidad de sobrevivir con ayuda de la medicina crítica son priorizados sobre los pacientes que tienen menor probabilidad de sobrevivir" 30 .

Asimismo, contempla para las personas que no califican para recibir cuidados críticos, que deben seguir recibiendo la atención hospitalaria para que se encuentren en la mejor situación posible al final de su vida, por lo que pueden beneficiarse de los cuidados paliativos. Requiere que

Si la medicación nos es suficiente para aliviar los síntomas se debe considerar la sedación, no con la intención de provocar la muerte pero si la intención de que el paciente termine su vida sin sufrimiento. La sedación paliativa en pacientes hipóxicos, en progresión de la enfermedad no subsidiaria al tratamiento, es considerada como una expresión de buena práctica clínica y debe seguir las recomendaciones existentes ${ }^{31}$.

La Guía también contempla que si existieran cambios en el estatus clínico del paciente y existiera disponibilidad de cuidados críticos, pudieran ser candidatos para ser admitidos. Recomienda también acompañar lo mejor posible al paciente en su proceso de muerte, facilitando la despedida entre él y su familia. Si no fuera posible que estén presentes por cuestiones sanitarias propone la Guía el uso de tecnología para hacer video-llamadas.

$$
\begin{aligned}
& { }^{27} \text { Ibid., p. } 5 . \\
& { }^{28} \text { Ibid. } \\
& { }^{29} \text { Ibid., p. } 6 . \\
& { }^{30} \text { Ibid., p. } 11 . \\
& { }^{31} \text { Ibid., p. } 13 .
\end{aligned}
$$




\section{CONCLUSIONES}

1. La inicial propuesta de la Guía Bioética para la asignación de los recursos limitados de medicina crítica en situación de emergencia, es discriminatoria por las siguientes razones:

a) Dicha guía viola diversos instrumentos internacionales en materia de Derechos Humanos, como los artículos 7 y 25.1 de la Declaración Universal de los Derechos Humanos; 24 y 29 de la Declaración Americana de los Derechos Humanos, el artículo 12.1 del Pacto Internacional de Derechos Económicos, Sociales y Culturales; artículo 26 del Pacto Internacional de los Derechos Civiles y Políticos; los puntos 24 y 31 de la Conferencia de Viena de 1982; los puntos 73, 74, 75, 75 y 90 del Plan de Acción Internacional de Madrid sobre el envejecimiento consecuencia de la Segunda Asamblea Mundial sobre el envejecimiento en Madrid, España del 8 al 12 de abril del 2002.

b) En el derecho interno de México, dicha guía viola los artículos 1, 4 y 73 fracción XVI de la Constitución Política de los estados Unidos Mexicanos; los artículos 5 fracción III y 18 de la Ley de los derechos de las Personas Adultas Mayores; el artículo 1bis, 2, 33 y 171 de la ley General de Salud; Artículo 4 de la Ley Federal para Prevenir y Eliminar la Discriminación. En El estado de Jalisco el artículo 6 de la Ley para el Desarrollo Integral del Adulto Mayor del Estado de Jalisco.

c) Es ilustrativa la definición de "Discriminación: Cualquier distinción, exclusión, restricción que tenga como objetivo o efecto anular o restringir el reconocimiento, goce o ejercicio en igualdad de condiciones de los derechos humanos y las libertades fundamentales en la esfera política, económica, social, cultural o en cualquier otra esfera de la vida pública y privada" ${ }^{\text {"2 }}$. En ella encontramos elementos de evidente exclusión del Adulto mayor, en el contenido de la mencionada Guía.

d) De igual forma la definición de "Discriminación por edad en la vejez": Cualquier distinción, exclusión o restricción basada en la edad que tenga como objetivo o efecto anular o restringir el reconocimiento, goce o ejercicio en igualdad de condiciones de los derechos humanos y libertades fundamentales en la esfera política, económica, social, cultural o en cualquier otra esfera de la vida pública y privada $^{33}$. De igual forma la primera propuesta de la Guía viola derechos tan fundamentales como la vida, la integridad física, la dignidad, el derecho a la salud, la igualdad, la no discriminación, etcétera.

e) Al establecer el principio adicional salvar la mayor cantidad de vidas por completarse, antepone de forma discriminatoria a las personas más jóvenes sobre los adultos mayores. Al prejuzgar que los adultos mayores ya no gozan de oportunidades valiosas al estar en una etapa avanzada de su vida, prejuzgando que gozan de mala salud y los jóvenes de buena salud.

f) Al establecer que ante iguales circunstancias se elija a los jóvenes deben ser atendidos preferentemente sobre los adultos mayores señalar como ya se mencionó

\footnotetext{
32 Art 2 Definiciones de la Convención Interamericana Sobre la Protección de los Derechos Humanos de las Personas Mayores, 2015. Disponible en http://www.oas.org/es/sla/ddi/docs/tratados_multilaterales_interamericanos_a70_derechos_humanos_personas_mayores.pdf. Es menester referir que México no ha firmado dicho instrumento internacional; sin embargo a sido exhortado por la CNDH, la Cámara de Diputados y la CONAPRED, para la ratificación del mismo.

${ }^{33}$ Ibid.
} 
en líneas anteriores se debe salvar la mayor cantidad de vidas por completarse, discriminando a los adultos mayores por el sólo hecho de serlo, sin atender a otras circunstancias al respecto, o la exclusión estructural de que son objeto la mayoría de los mexicanos y mexicanas, que ha implicado la afectación a su calidad de vida al llegar a la edad de adultos mayores.

2. La aprobación finalmente de la última versión de la Guía Bioética para asignación de recursos limitados de medicina crítica en situación de emergencia, implicó un cambio sustantivo con respecto a la primera propuesta:

a) Incorporó la mayoría de los criterios esgrimidos por la CNDH, la CONAPRED, la Cámara de diputados federal, entre otros los fundamentos de igualdad y no discriminación, el derecho a la salud, el enfoque humanista y el respeto a los principios bioéticos en el tenor del respeto a la integridad, la salud y la dignidad de las personas. Los principios de la bioética: beneficencia, no maleficencia, autonomía y justicia.

b) Excluyó expresamente cualquier criterio de discriminación por la edad del paciente y estableció que la característica que debe tomarse en cuenta en la asignación de recursos escasos de medicina crítica es que se pueda beneficiarse de dichos recursos médicos.

c) En la Guía aprobada refiere que la edad cronológica o la discapacidad, no son características que excluyan para recibir cuidados críticos. Por lo que considera un error asumir que la edad o discapacidad, es sinónimo de vida inferior o mala salud.

d) Esta versión vigente de la Guía respeta la autonomía del paciente, mediante el pleno ejercicio del derecho a la información; la voluntad anticipada,, el respeto del paciente a no ser sujeto de un procedimiento médico; y en su caso, recibir cuidados paliativos.

e) El criterio de la Guía es salvar la mayor cantidad de vidas de los pacientes, por lo que los pacientes que según el diagnóstico tienen mayor posibilidad de vivir recibirán los recursos escasos de medicina crítica. Debiendo ser revaluadas las personas que inicialmente no se beneficiaron si se encuentran en una mejor situación, o mejora.

f) La Guía tiene un enfoque humanista al contemplar que el paciente pueda terminar la vida sin sufrimiento a través de la sedación paliativa, misma que se considera una buena práctica clínica. Recomienda acompañar al paciente y facilitar en su caso, la despedida de éste con sus familiares o amigos a través de los medios tecnológicos al alcance, como video-llamadas.

3. Asumir la primera versión de la Guía Bioética para la asignación de los recursos limitados de medicina crítica en situación de emergencia, implicaría un serio retroceso a la convicción de que las personas tenemos derechos y que los mismos deben de ser garantizados por el Estado y por el conjunto de las instituciones que le conforman e integran, como es el caso del Consejo de Salubridad General. Los derechos humanos y la dignidad de las personas mayores, no deben ser objeto de minusvaloración por el establecimiento de criterios tales como "vidas por completarse", si efectivamente atendemos a la convicción del igual valor y respeto por las personas, con independencia de cualquier condición o circunstancia en la que se encuentren en los términos del artículo $1^{\circ}$ de la Constitución Mexicana. La Guía actualmente aprobada no es perfecta, no contempla la totalidad de las recomendaciones realizadas por las instituciones; pero 
implica, la rectificación y apertura de miras, de cara a la solidaridad colectiva y fraternal. La confianza de los ciudadanos hacia las autoridades especialmente en materia sanitaria; es quizá, el mayor beneficio obtenido como resultado de esta colisión de posturas bioéticas respecto el contenido y alcance de los derechos de los adultos mayores a recibir atención médica en el contexto de la emergencia sanitaria de COVID19 en México.

4. Cabe destacar que era necesaria la creación de una Guía Bioética de México, sin embargo, su contenido inicial fue preocupante, denotando una clara falta de humanismo en las políticas públicas de la presente administración. Es de gran relevancia observar la oportuna intervención de la Comisión de Derechos Humanos y de la Cámara de Diputados intentando encontrar un equilibrio. Es plausible que se logró modificar la Guía Bioética primando la igualdad y no discriminación, sin embargo, es evidente que el Consejo de Salubridad General del gobierno federal mexicano tuvo una reacción tardía, poco transparente y primando decisiones diversas a la salud pública. En este sentido se explica las medidas desesperadas de pretender la aprobación de un instrumento como la primera Guía Bioética sin incluir derechos humanos fundamentales como el de la igualdad y la del derecho a la vida de los adultos mayores.

\section{REFERENCIAS BIBLIOGRÁFICAS}

- AURENQUE, D., "Consideraciones éticas en el contexto de triaje por COVID-19", Rev. Méd. Chile, núm. 148, pp. 557-564.

- DÍAZ, T., "Aspectos internacionales del derecho a la salud", Saber, Ciencia y Libertad, 2010, pp. 51-60.

- DODDOLI, C., "Coronavirus ¿Qué es la guía bioética para pacientes con COVID-19?”, Ciencia-UNAM, 18 de mayo de 2020. Disponible en http://ciencia.unam.mx/leer/997/coronavirus-que-es-la-guia-bioetica-para-pacientes-concovid-19-

- MARKMANN, G., ET AL. "Possibilities and limits of institutional ethics services in response to the COVID-19 pandemic", Akademie für Ethik in der Medizin, 31 de marzo de 2020. Disponible en https://repository.publisso.de/resource/frl:6419971/data

- MIRANDA, P., "Bajan a "proyecto" la Guía Bioética para asignar recursos durante Covid19”, El Universal, 16 de abril de 2020. Disponible en https://www.eluniversal.com.mx/nacion/bajan-proyecto-la-guia-bioetica-para-asignarrecursos-durante-covid-19

- MORENO, T., "UNAM se deslinda de guía que recomienda priorizar a jóvenes sobre tercera edad", El Universal, 14 de abril de 2020. Disponible en https://www.eluniversal.com.mx/nacion/coronavirus-unam-se-deslinda-de-guia-querecomienda-priorizar-jovenes-sobre-tercera-edad

- MORENO, T., "Guía Bioética, "inadecuada" ante contingencia por Covid-19: experto de la UNAM", El Universal, 15 de abril de 2020. Disponible en https://www.eluniversal.com.mx/nacion/guia-bioetica-inadecuada-ante-contingencia-porcovid-19-experto-de-la-unam 
- MONZÓN MARÍN, J. L., COUCEIRO VIDAL, A., "Algunas consideraciones sobre la ética del triaje en UCI durante la pandemia COVID-19", Medicina Intensiva, 2020 (en prensa). https://doi.org/10.1016/j.medin.2020.06.008

- POY, L., "Manuel Ruiz de Chávez dimite como comisionado nacional de Bioética", La Jornada, 16 abril de $2020 . \quad$ Disponible en https://www.jornada.com.mx/ultimas/politica/2020/04/16/renuncia-el-comisionadonacional-de-bioetica-manuel-ruiz-de-chavez-9295.html

- REDACCIÓN AN, "Consejo de Salubridad General no aprobó "Guía bioética"; es un borrador", Aristegui Noticias, 15 de abril de 2020. Disponible en https://aristeguinoticias.com/1504/mexico/consejo-de-salubridad-general-no-aprobo-guiabioetica-es-un-borrador-enterate/

- RUEDA, J., "¿No es país para viejos? La edad como criterio de triaje durante la pandemia COVID-19”, Enrahonar, núm. 65, 2020, pp 85-98. DOI: 10.5565/rev/enrahonar.1306

- SÁNCHEZ, A., "Guía Bioética de Medicina Crítica debe ser ampliamente discutida: UNAM", La Jornada, 15 de abril de 2020. Disponible en https://www.jornada.com.mx/ultimas/sociedad/2020/04/15/guia-bioetica-de-medicinacritica-debe-ser-ampliamente-discutida-unam-471.html

- SEEDHOUSE, D., "Why bioethicists have nothing useful to say about health care rationing", Journal Medical ethics, vol. 21, núm. 5, 1995, pp 288-291. DOI: 10.1136/jme.21.5.288

- TORIBIO, L. y RODRÍGUEZ, P., "Renuncia delegado de Guía de Bioética de COVID-19", Imagen Radio, 17 de abril 2020. Disponible en https://www.imagenradio.com.mx/renunciadelegado-de-guia-de-bioetica-de-covid-19

\section{Documentos}

- ACADEMIA SVIZZERA DELLE SCIENZE MEDICHE, Pandemia Covid-19: triage dei trattamenti di medicina intensive in caso di scarsità di risorse. Indicazioni per làttuazione de capitol 9.3 delle directive dell'ASSAM Provvedimenti di medicina intensive (2013).

- COMISIÓN INTERAMERICANA DE LOS DERECHOS HUMANOS, La CIDH urge a los Estados a garantizar los derechos de las personas mayores frente a la pandemia del COVID-19, 23 de abril de 2020. Disponible en http://www.oas.org/es/cidh/prensa/comunicados/2020/088.asp

- COMISIÓN INTERAMERICANA DE LOS DERECHOS HUMANOS, Resolución no. 1/2020 pandemia y derechos humanos en las Américas, 10 de abril de 2020. Disponible en (https://www.oas.org/es/cidh/decisiones/pdf/Resolucion-1-20-es.pdf

- COMISIÓN NACIONAL DE DERECHOS HUMANOS, La Comisión Nacional de los Derechos Humanos exhorta al Consejo de Salubridad General a implementar medidas para garantizar los derechos humanos de todas las mexicanas y mexicanos y rechazar medidas discriminatorias, con motivo de la emergencia derivada de la pandemia originada por el coronavirus COVID-19, 17 de abril de 2020. Disponible en https://www.cndh.org.mx/documento/la-comision-nacional-de-los-derechos-humanosexhorta-al-consejo-de-salubridadgeneral\#: : text=La\%20CNDH\%20exhort\%C3\%B3\%20al\%20Consejo,por\%20el\%20coron avirus\%20COVID\%2D19 
- CONBIOÉTICA, Recomendaciones Bioéticas ante el COVID-19, 31 de marzo de 2020. https://www.gob.mx/cms/uploads/attachment/file/546480/RECOMENDACIONES_BIOET ICAS ANTE EL COVID-19 Final.pdf

- CONBIOÉTICA, La Bioética ante la pandemia del COVID-19, 12 de marzo de 2020. https://www.gob.mx/cms/uploads/attachment/file/544215/Pronunciamiento_Bioetica_ante_1 a pandemia del COVID 19.pdf

- CONSEJO DE SALUBRIDAD GENERAL, Guía bioética para asignación de recursos limitados de medicina crítica en situación de emergencia (Versión actual, 30 de abril de 2020). Disponible

en http://www.csg.gob.mx/descargas/pdf/index/informacion_relevante/GuiaBioeticaTriaje_30_ Abril $20207 \mathrm{pm} . \mathrm{pdf}$

- CONSEJO DE SALUBRIDAD GENERAL, Guía bioética para asignación de recursos limitados de medicina crítica en situación de emergencia (Primera versión, 13 de abril de 2020). Disponible en https://interactivo.eluniversal.com.mx/online/pdf-20/PDFGUIA_Bioetica_FINAL_13_Abril2020.pdf

- CONSEJO NACIONAL PARA PREVENIR LA DISCRIMINACIÓN (CONAPRED), El impacto de la pandemia de COVID-19 en los grupos discriminados y alternativas de atención, Ciudad de México, 8 de mayo de 2020. Disponible en http://www.conapred.org.mx/index.php?contenido=boletin\&id=1351\&id_opcion=103\&op= $\underline{213}$

- CONSEJO NACIONAL PARA PREVENIR LA DISCRIMINACIÓN (CONAPRED), Diez preguntas frecuentes sobre discriminación y COVID-19, 5 de mayo de 2020. Disponible en http://www.conapred.org.mx/userfiles/files/Diez_Preguntas_COVID-19_5-05-2020.pdf

- CONSTITUCIÓN POLÍTICA DE LOS ESTADOS UNIDOS MEXICANOS.

- CORTE INTERAMERICANA DE LOS DERECHOS HUMANOS, Declaración de la Corte Interamericana de Derechos Humanos 1/20. COVID-19 y Derechos Humanos: Los problemas y desafios deben ser abordados con perspectivas de Derechos Humanos y Respetando las obligaciones Internacionales, 9 de abril de 2020. Disponible en https://www.corteidh.or.cr/tablas/alerta/comunicado/declaracion_1_20_ESP.pdf

- OFICINA DEL ALTO COMISIONADO DE LAS NACIONES UNIDAS PARA LOS DERECHOS HUMANOS, Las medidas de emergencia y el COVID-19: Orientaciones, 27 de abril de 2020.2 Disponible en https://www.ohchr.org/Documents/Events/EmergencyMeasures_COVID19_ES.pdf

- ORGANIZACIÓN MUNDIAL DE LA SALUD, Alocución de apertura del Director General de la OMS en la rueda de prensa sobre la COVID-19 celebrada el 11 de marzo de 2020. Disponible en https://www.who.int/es/director-general/speeches/detail/who-directorgeneral-s-opening-remarks-at-the-media-briefing-on-covid-19---11-march-2020 11 de $\underline{\text { marzo } 2020}$

- Principios de Siracusa. https://undocs.org/pdf?symbol=es/E/CN.4/1985/4

- Proposición con punto de acuerdo por la que la Cámara de Diputados del H. Congreso de la Unión rechaza que la Guía bioética de asignación de recursos de medicina crítica, se establezca cualquier tipo de criterio basado en la edad o cualquier tipo de discriminación, para la atención crítica de los enfermos por COVID-19. Disponible en http://gaceta.diputados.gob.mx/PDF/64/2020/abr/PROPOS-28-ABR/Propo-Morena-27.pdf 
- SECRETARÍA DE SALUD, Se presentará propuesta de Guía para la asignación de recursos de contingencia a vocales del CSG, 16 de abril de 2020. Disponible en http://www.csg.gob.mx/descargas/triaje/Comunicado_108 CSG Guixa Asignacioxn recur sos_en_situacioxn_de_contingencia150420.pdf

- SECRETARÍA DE SALUD, Se declara en sesión permanente el Consejo de Salubridad General, 19 de marzo de 2020. Disponible en https://www.gob.mx/salud/prensa/092-sedeclara-en-sesion-permanente-el-consejo-de-salubridad-general 